\title{
Aspectos Anatómicos y Biométricos de los Puentes de Miocardio y sus Relaciones con la Arteria Interventricular Anterior y Venas Adyacentes
}

\author{
Anatomical and Biometric Aspects of the Myocardial Bridges and its Relationships \\ with the Anterior Interventricular Artery and Adjacent Veins
}

"Sousa-Rodrigues, C.F.; "Soares de Alcantara, F.; "Buarque de Gusmão, L.C.; "da Rocha, A. C.; Alencar e Silva, R. \& ** Olave, E.

SOUSA-RODRIGUES, C. F.; ALCANTARA, S. F.; BUARQUE, G. L. C.; ROCHA, A. C.; ALENCAR E SILVA, R. \& OLAVE. E. Aspectos anatómicos y biométricos de los puentes de miocardio y sus relaciones con la arteria interventricular anterior y venas adyacentes. Int. J. Morphol., 24(2):279-284, 2006.

RESUMEN: Los vasos cardíacos pueden estar parcialmente cubiertos por puentes de miocardio, disposición que ha sido objeto de numerosos estudios. El objetivo del presente trabajo fue determinar la incidencia de estos puentes, su extensión y sobre que vasos se disponen, considerando principalmente las relaciones con los vasos interventriculares anteriores. El material estudiado fueron 30 corazones de individuos adultos, brasileños, fijados en formaldehído al $10 \%$. Se encontraron puentes de miocardio en 14 muestras (47 \%) y de éstos, en 9 se localizaron por sobre la arteria interventricular anterior; en 3 casos se ubicaron simultáneamente sobre la arteria mencionada y sobre afluentes de la vena homónima; en 1 corazón puentes de miocardio se presentaron por sobre ramas ventriculares izquierdas de la arteria y vena interventricular posterior, como también en 1 muestra estuvo por sobre una rama ventricular anterior de la arteria coronaria derecha. Los puentes se localizaron principalmente en la parte proximal y media del surco interventricular anterior. La extensión de los puentes encontrados varió entre 9 y $55 \mathrm{~mm}$, con un promedio de 24,5. De acuerdo a los resultados obtenidos la incidencia de estas formaciones musculares es alta, localizándose generalmente sobre la arteria interventricular anterior y en porcentajes menores sobre venas adyacentes.

PALABRAS CLAVE: Anatomía; Angiología; Corazón; Puentes de miocardio.

\section{INTRODUCCIÓN}

Es conocido el hecho que los vasos cardíacos pueden estar parcialmente cubiertos por puentes de miocardio, lo que ha sido motivo de numerosos estudios. Estos puentes son formaciones musculares que se originan desde el miocardio ventricular y se posicionan por sobre estructuras vasculares del corazón y pueden ser vistos después de retirar el epicardio y el tejido graso subepicárdico (James, 1961; Baroldi \& Giuseppe, 1965).

Según DiDio (2002) ya en los inicios del Siglo XX esta formaciones fueron descritas en mamíferos domésticos y en primates superiores. Los puentes de miocardio en humanos fueron considerados inicialmente como simples curiosidades anatómicas, los que luego pasaron a ser foco de atención de los investigadores, despertando el interés clínico y quirúrgico.
Los estudios en seres humanos vivos pasaron a ser realizados a través de angiografías, cuando a partir de 1960 se observó el estrechamiento sistólico de la rama interventricular anterior de la arteria coronaria izquierda ( Angelini et al., 1983).

Los puentes de miocardio son un hallazgo frecuente y su incidencia depende de los métodos utilizados para su estudio. Así, los porcentajes informados son bajos cuando se utilizan métodos angiográficos y mucho más altos cuando se realizan estudios en necropsias.

Baptista et al. (1992) señalaron que los puentes de miocardio son más frecuentes que ausentes y más frecuentemente por sobre la rama interventricular anterior de la arteria coronaria izquierda, donde son también de mayor extensión.

\footnotetext{
* Universidad Federal de Alagoas, Maceió, Brasil
}

*** Universidad de La Frontera, Temuco, Chile. 
Estos autores notaron que el ángulo entre la dirección de los fascículos de miocardio "pontino" y de la arteria "subpontina" varía de 1 (casi paralela) a 90 grados (perpendicular), siendo éste último valor el más frecuentemente encontrado.

Por su parte Ortale et al. (2001) encontraron una mayor incidencia de puentes sobre la arteria interventricular anterior. Estos mismos autores señalaron que la extensión de los puentes, estando sobre arterias o venas es variable.

Con relación a las venas cardíacas, Pejkovich \& Bogdanovic (1992) citaron que están parcialmente cubiertas por estos puentes sólo en algunas ocasiones, acrecentando que cuando no cubren propiamente a la vena interventricular anterior y solamente lo hacen sobre sus tributarias menores, éstos pueden ser causa de alteración en el retorno venoso de la región.

El objetivo de la presente investigación fue de estudiar la incidencia de los puentes de miocardio en individuos brasileños, su localización, el o los vaso (s) sobre el que preferentemente se disponen, así como la extensión de los mismos y su relación con la rama interventricular anterior de la arteria coronaria izquierda y venas adyacentes, sabiendo que estos parámetros pueden ser de importancia para la anatomía cardiaca.

\section{MATERIAL Y MÉTODO}

El material utilizado fueron 30 corazones de individuos adultos, de ambos sexos, fijados en solución de formaldehído al 10\%, disponibles para esta investigación en los laboratorios de Anatomía Topográfica del Centro de Ciencias Biológicas de la Universidad Federal de Alagoas (UFAL) y de la Universidad de Ciencias de la Salud (UNCISAL), Brasil.

La disección fue realizada con material ad hoc, removiendo el epicardio y el tejido graso subepicárdico, evidenciando los vasos y formaciones musculares en estudio.

Se verificó la presencia de los puentes de miocardio, así como las arterias y venas cubiertas por los mismos. Para las mediciones se usó un caliper mecánico manual (Metrica).

\section{RESULTADOS}

Los puentes de miocardio se presentaron en 14 de los 30 corazones $(46,7 \%)$, de los cuales, en 12 (40\%) se localizaron sobre el surco interventricular anterior; en uno $(3,3 \%)$, el puente estaba sobre la superficie diafragmática o inferior del ventrículo derecho y en el otro caso $(3,3 \%)$, estaba en la cara anterior del ventrículo derecho.

De los 12 casos en que los puentes se localizaron sobre el surco interventricular anterior, en 8 de ellos $(26,7 \%)$ se ubicaron sobre su tercio medio; en 3 (10\%) estuvieron sobre el tercio superior del mismo surco y en un caso $(3,3 \%)$ se extendió desde el tercio superior al inferior del surco mencionado.

En relación a los vasos sobre los cuales los puentes se dispusieron e el surco interventricular anterior, en los 12 casos lo hicieron sobre la arteria interventricular anterior, de los cuales en $9(30 \%)$ se localizaron exclusivamente sobre la misma y en 3 casos (10\%), simultáneamente sobre esta última y afluentes de la vena interventricular anterior (Fig.1). No se encontró puente de miocardio sobre la vena mencionada anteriormente.

Respecto a los puentes de miocardio ubicados exclusivamente sobre la arteria interventricular anterior (9 casos), en 5 corazones $(17 \%)$ ocupó el tercio medio del surco interventricular anterior (Fig. 2); en 3 (10\%) estuvo sobre el tercio superior del mismo surco y en uno (1\%) se extendió desde el tercio superior al inferior del surco interventricular anterior (Fig. 3).

En los casos en que el puente de miocardio estuvo simultáneamente sobre la arteria interventricular anterior y afluentes de la vena homónima (3 corazones), su disposición fue sólo en el tercio medio del surco interventricular anterior.

En el caso (3\%) en que el puente de miocardio fue encontrado en la superficie diafragmática del ventrículo derecho, se dispuso sobre ramas y afluentes ventriculares derechos de la arteria y vena interventriculares posteriores, respectivamente.

En el caso (3\%) en que el puente de miocardio estuvo sobre la cara anterior del ventrículo derecho, se extendió por sobre una rama interventricular anterior de la arteria coronaria derecha.

Los registros métricos de la extensión de los puentes de miocardio variaron entre 9 y $55 \mathrm{~mm}$, con um promedio de $24,5 \mathrm{~mm}$. El lugar donde tuvieron mayor extensión fue sobre el surco interventricular anterior (12 a 55 $\mathrm{mm}$ con un promedio de $26,4 \mathrm{~mm}$ ). En la superficie diafragmática del ventrículo derecho, el puente tuvo una extensión de $11 \mathrm{~mm}$ y el de la cara anterior del ventrículo derecho tuvo $9 \mathrm{~mm}$. 

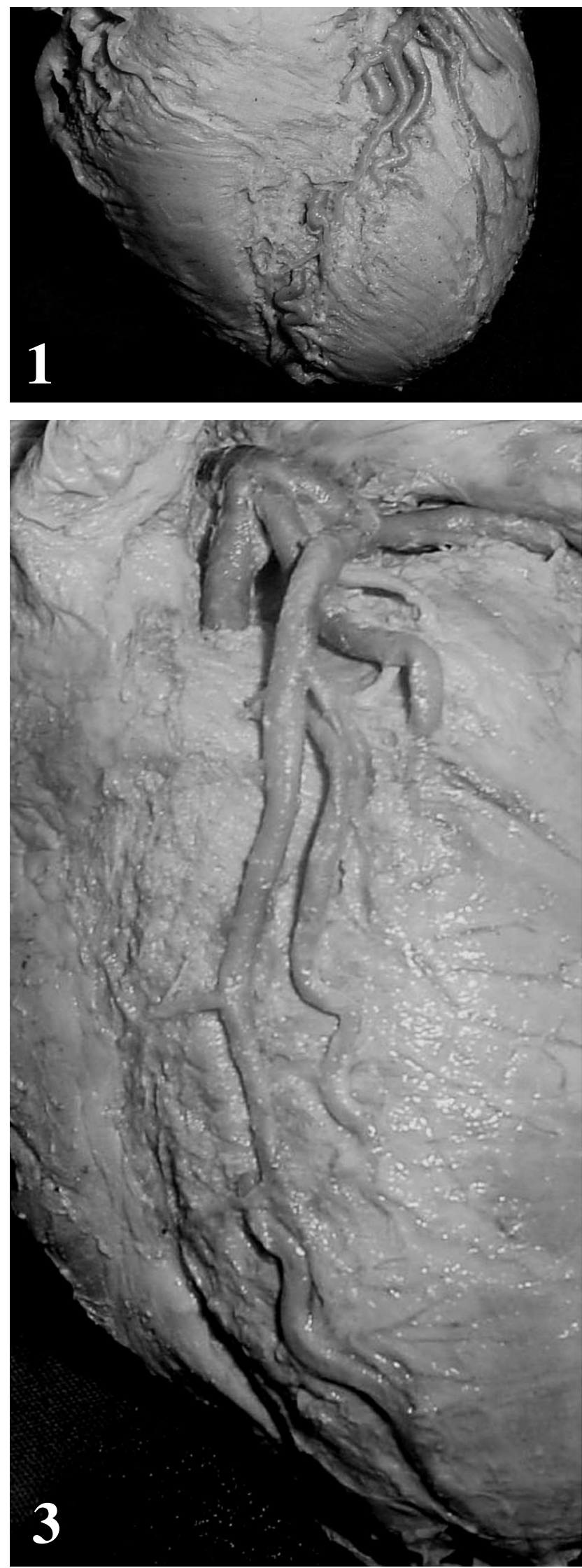

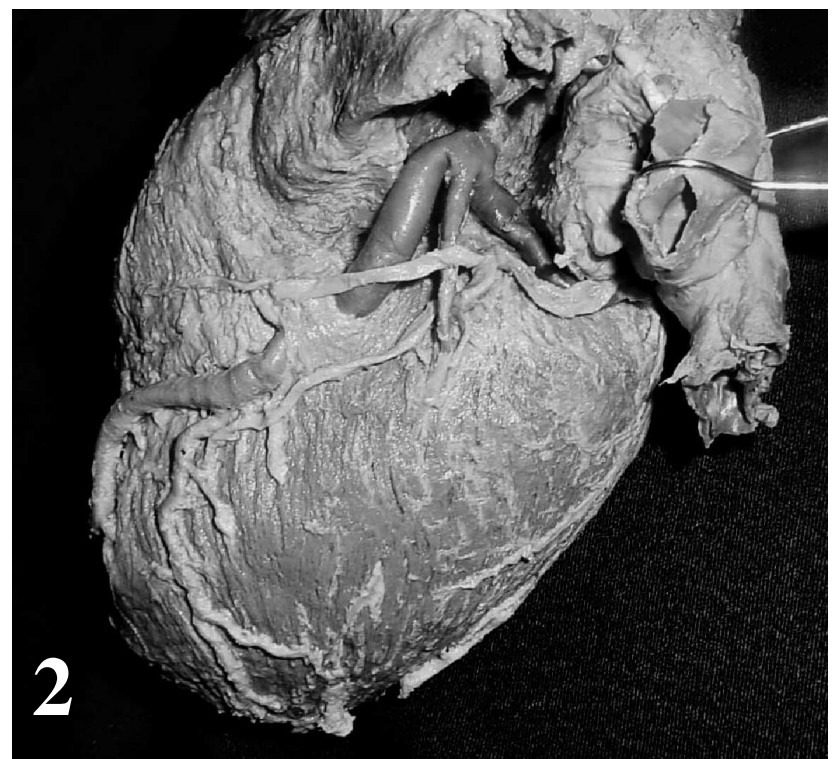

Fig. 1. Puente de miocardio sobre la rama interventricular anterior de la arteria coronaria izquierda y afluente ventricular derecho de la vena interventricular anterior.

Fig. 2. Puente de miocardio de $12 \mathrm{~mm}$ sobre la parte media de la rama interventricular anterior de la arteria coronaria izquierda.

Fig. 3. Puente de miocardio de $55 \mathrm{~mm}$ sobre la rama interventricular anterior.

\section{DISCUSIÓN}

La posición subepicárdica de las arterias coronarias es de conocimiento general, sin embargo, con cierta frecuencia algunas arterias se introducen y recorren un cierto trayecto bajo una capa de fibras musculares cardíacas y después reaparecen en la superficie. A estas formaciones musculares se le conocen como puentes de miocardio.

A esta disposición se le ha denominado de diversas maneras. Considerando a las arterias, Edwards et al. (1956) las denominaron como "intramurales"; Geiringer (1951) y Morales et al. (1980) como “intraparietales'.

Otros autores se basaron en las fibras musculares y de ahí el término de puentes de miocardio (Polacek, 1959; Furtado et al., 1979). 
Estos puentes de miocardio son un hallazgo relativamente frecuente y han sido relacionado con ciertas patologías cardíacas. En muchos casos cursan de forma asintomática, sin embargo, su presencia ha sido considerada como causa de angina, arritmias malignas, infartos de miocardio y muerte súbita (Arnau et al., 1999). Estos autores presentaron un caso de isquemia aguda provocada por un puente de miocardio sobre la rama interventricular anterior.

Mientras que en corazones autopsiados su presencia varía de 15 a $85 \%$, con métodos angiográficos se ha reportado entre 0,5 y $2,5 \%$ de los casos (Okmen et al., 2002). Por su parte, Arnau et al., también señalaron que habían amplias diferencias entre estudios en necropsias y los angiográficos, explicando que la diferencia se debería a que no todos los puentes musculares producen compresión sistólica, al menos detectable, y agregado a ello, está la oblicuidad y/o profundidad de sus fibras.

La frecuencia encontrada en nuestra serie es levemente inferior al 53\% encontrado por Baptista et al. (1992), sin embargo, nuestros resultados corroboran lo expresado por estos autores cuando señalaron que estos puentes se encuentran más frecuentemente sobre la arteria interventricular anterior,

La presencia de puentes de miocardio en corazones de individuos chilenos fue señalada por Henríquez et al. (1986) en un $88 \%$ de los casos, así como Bezerra et al. (1987) que los encontraron en 39 de 50 corazones brasileños $(78 \%)$, valores superiores al de nuestra serie,. Por otra parte, valores menores han sido reportados por Venkateshu et al., 2000 (14,2\% en 106 casos); Kosinski \& Grzybiak, 2001 (41\% en 100); Kosinski et al., 2004 (31,3\% en 300 corazones), entre otros.

Sobre la arteria o rama que más frecuentemente se localizaron estos puentes, la interventricular anterior es la más mencionada. Así, Henríquez et al. señalaron que 31 $(73,8 \%)$ de los 42 puentes que encontraron se ubicaron sobre la arteria coronaria izquierda y sus ramas, de los cuales, $25(59,5 \%)$ estuvieron sobre la arteria interventricular anterior, siendo semejante al resultado obtenido por Ortale $e t$ $a l$, quienes señalaron un $56 \%$. Tales resultados son menores que los encontrados en nuestro trabajo, ya que $12(85,7 \%)$ de los 14 corazones que tenían los puentes de miocardio, se localizaron por sobre la arteria interventricular anterior, de los cuales en 9 fue exclusivamente sobre ella, los otros tres casos también involucraron a tributarias de la vena interventricular anterior.

También Bezerra et al. mencionaron que $26(66,7 \%)$ de los 39 corazones con puentes estuvieron sobre la arteria mencionada. Otros autores corroboraron esta disposición (Venkateshu et al.; Kosinski \& Grzybiak; Okmen et al.; Kosinski et al.).

Otras arterias sobre las cuales se localizan estos puentes, pero en porcentajes menores, es sobre la arteria marginal izquierda, rama posterior del ventrículo derecho, arteria diagonal, (Henríquez et al.; Bezerra et al; Venkateshu et al.; Kosinski et al.).

Puentes sobre la arteria coronaria derecha son raros y cuando se presentan lo hacen sobre la rama interventricular posterior (Okmen et al.). Estos mismos autores señalaron que puentes sobre la arteria circunfleja se encuentran muy ocasionalmente, presentando ellos mismos, un puente de aproximadamente $2,5 \mathrm{~cm}$ sobre esta arteria, la que descubrieron a través de una arteriografía.

En nuestro estudio, cuando los puentes se localizaron únicamente sobre la arteria interventricular anterior, éstos se dispusieron principalmente en la parte media y en la parte proximal del surco homónimo, concordando con lo descrito por Lima et al. y Kosinski et al.

Con respecto a la longitud de los puentes, registramos valores entre 9,0 y $55 \mathrm{~mm}$ con un promedio de 24,5 $\mathrm{mm}$.,datos concordantes con el promedio de $25 \mathrm{~mm}(6,25 \mathrm{a}$ $63,12 \mathrm{~mm}$ ) de Lima et al., siendo levemente superiores a los de Henríquez et al. cuyos valores fueron entre 1,9 y 47,9 $\mathrm{mm}$ y promedio de $15,0 \mathrm{~mm}$.

Nuestros datos son también concordantes con los de Ortale et al. localizados entre 3 y $50 \mathrm{~mm}$ y Kosinski et al. con valores entre 2,3 y 42,8 mm, diferentes de los informados por Pejkovic \& Bogdanovic que sólo los encontraron con extensiones entre 2 y $10 \mathrm{~mm}$. y de Venkateshu et al. que indicaron que la mayoría de los puentes de su estudio tenían entre 10 y $19 \mathrm{~mm}$.

Con relación a los puentes de miocardio sobre afluentes de la vena interventricular anterior, nuestros resultados (10\%) muestran discordancia respecto a los informados por Ortale et al. (5\%) y por Pejkovich \& Bogdanovich (2\%). Estas formaciones musculares pueden al menos interrumpir o disminuir el flujo sanguíneo en las venas durante el sístole ventricular (von Lüdinghausen, 1987).

Desde este punto de vista, es importante considerar que los puentes de miocardio no sólo cubren a las arterias sino que también lo hacen sobre algunas venas, de paredes más delgadas, lo que podría repercutir en el retorno venoso cardíaco. 
SOUSA-RODRIGUES, C. F.; ALCANTARA, S. F.; BUARQUE, G. L. C.; ROCHA, A. C.; ALENCAR E SILVA, R. \& OLAVE. E. Anatomical and biometric aspects of the myocardial bridges and its relationships with the anterior interventricular artery and adjacent veins. Int. J. Morphol., 24(2):279-84, 2006.

SUMMARY: The cardiac vessels are sometimes partly overlain by myocardial bridges, what has been subject of study by innumerous researchers. The present work has as objective to study the incidence of the myocardial bridges, its length and above which vessels they are preferencially. The material consisted of 30 hearts of adult individuals of both sexes, fixed in 10\% formaldeyde solution. Myocardial bridges were found in 14 hearts (47\%); about these, there were in 9 hearts (30\%) muscle bridges only above the anterior interventricular artery; in 3 cases (10\%) simultaniously over anterior interventricular artery and afluents of the anterior interventricular vein; in 1 heart (3\%), it ocurred over left ventricular branches of the posterior interventricular artery and vein and in 1 heart (3\%) over anterior ventricular branch of the right coronary artery. The length of the muscle bridges ranged from 9 to $55 \mathrm{~mm}$. (mean $24.5 \mathrm{~mm}$ ). We concluded that the incidence of the myocardial bridges is high and the vessel above which they are formed in greater frequency was the anterior interventricular artery in its middle portion and in minor percentages on adjacent veins .

\section{KEY WORDS: Anatomy; Angiology; Heart; Myocardial bridges}

\section{REFERENCIAS BIBLIOGRÁFICAS}

Angelini, P.; Trivellato, M.; Donis, J.; Leachman, R. D. Myocardial bridge: a review. Prog. Cardiovasc. Dis., 26: $75-88,1983$

Arnau, M. A.; Martínez, L.V.; Almenar, L.; Lalaguna, L.; Ten, F. \& Palencia, M. Puente miocárdico como causa de isquemia aguda. Descripción del caso y revisión de la bibliografía. Rev. Esp. Cardiol., 52:441-4, 1999.

Baptista et al. (1992) Apud Didio, L. A. Sinopse de Anatomia Humana. $1^{\circ}$ ed. Rio de Janeiro, Guanabara-Koogan, 2002.

Baroldi, G. \& Giuseppe, S. Coronary Circulation in the normal and the pathologic heart. $1^{\circ}$ ed. Washington, Armed Forces Institute of Pathology, 1965.

Bezerra, A.; Didio, J. A. \& Prates, J. C. Pontes de miocardio. An. Anat. Norm., 5:59-65, 1987

Didio, L. A. Sinopse de Anatomia Humana. $1^{\circ}$ ed. Rio de Janeiro, Guanabara-Koogan, 2002.

Edwards, J. C.; Burnsides, C.; Swarm, R. L. \& Lansing, A. I. Arteriosclerosis in the intramural and extramural portion of coronary arteries in the human Herat. Circulation, 13(2):235-41, 1956.

Furtado, R. J.; Souza, J. E.; Thom, A. F.; Martins, L. R. F.; Pimentel, W. A.; Meneghello, R. S.; Ribeiro, W.; Lessa, I. S.; Forte, A. \& Zorzo, D. Cintilografía miocárdica com Thallium-201 em pacientes com ponte miocárdica na artéria descendente anterior. Arq. Bras. Cardiol., 32:160, 1979.

Geiringer, E. The mural coronary. Am. Heart. J., 41:359368,1951

Henríquez, J.; Olave, E.; Escobar, J. \& Monje, N. Puentes de miocardio sobre arterias coronarias en individuos chilenos. An. Anat. Nor., 4:53-57, 1986.

James, T. N. Anatomy of the Coronary Arteries. $1^{\circ}$ ed. New York, Medical Division of Harper \& Brothers, 1961.

Kosinski, A. \& Grzybiak, M. Myocardial bridges in the human heart: morphological aspects. Folia Morphol. (Warsz), 60(1):65-8, 2001.

Kosinski, A.; Grzybiak, M.; Skwarek, M. \& Hreczecha, J. Distribution muscular bridges in the adult human heart. Folia Morphol. (Warsz), 63(4):491-8, 2004

Lüdinghausen von, M.V. Clinical Anatomy of the Cardiac Veins. Surg. Radiol. Anat., 9:159-68, 1987

Lima, V. J. M.; Cavalcanti, J. S. \& Tashiro, T. Pontes de Miocárdio e sua Relação com o Ramo Interventricular Anterior da Artéria Coronária Esquerda. Arq. Bras. Cardiol., 79:215-8, 2002

Morales, A. R.; Romanelli, R. \& Boucek, R. J. The mural left anterior descending coronary artery, strenuous exercise and suden death. Circulation, 62(2):230-7, 1980. 
Okmen, E.; Oguz, E.; Erdinler, I.; Sanli, A. \& Cam, N. Left circumflex coronary artery bridging. Jpn. Heart J., 43:423-7, 2002

Ortale JR, Gabriel EA, Iost C, Màrquez CQ. The Anatomy of the Coronary Sinus and its Tributaries. Surg. Radiol. Anat., 23:15-21, 2001.

Pejkovich, B \& Bogdanovich, D. The Great Cardiac Vein. Surg. Radiol. Anat., 14:23-8, 1992.

Polacek, P. Muscular bridges and loops on coronary arteries of man. Cs. Morfol.,7(2):119-25, 1959.

Venkateshu, K.V.; Mysorekar, V.R.; Sanikop, M.B. Myocardial bridges. J. Indian Med. Assoc., 98(11):6913, 2000.
Dirección para Correspondencia: Prof. Dr. Célio de Sousa-Rodrigues Disciplina de Anatomía Topográfica Departamento de Morfología-Centro de Ciencias Biologicas-UlFAL Praça Afranio Jorge, s/no Prado CEP 57010-060

Maceió, Alagoas BRASIL

Email: cfrs2@aol.com

Recibido : 06-01-2006

Aceptado: 03-05-2006 